\title{
Basigin/CD147 Promotes the Activation of Signal Transduction Mediated by MyD88 and TRIF
}

\author{
Jieun Jeong ${ }^{1}$, Eunhee G. Kim ${ }^{1}$, Jae Y. Cho ${ }^{2}$, Eugene C. $\mathrm{Yi}^{3^{*}}$ and Kristine M. Kim ${ }^{1,4^{* *}}$ \\ ${ }^{1}$ Department of Systems Immunology, College of Biomedical Science, Kangwon \\ National University, Chuncheon 200-701, Korea \\ ${ }^{2}$ Department of Genetic Engineering, Sungkyunkwan University, Suwon 440-746, \\ Korea \\ ${ }^{3}$ Department of Molecular Medicine and Biopharmaceutical Sciences, Seoul National \\ University, Seoul, Korea \\ ${ }^{4}$ Institute of Bioscience and Biotechnology, Kangwon National University, \\ Chuncheon, 200-701, Korea \\ *euyi@snu.ac.kr,**kmkim@kangwon.ac.kr
}

\begin{abstract}
Basigin/EMMPRIN/CD147 has been reported to be associated with inflammatory diseases and cancers. Detailed insight into this multifunctional protein in the context of cellular functions may provide a key to its pathophysiological role in many diseases. Here, we investigated the potential role of basigin in activation of the $N F-\kappa B$ and AP-1 signaling cascades associated with the MyD88 and TRIF adaptor proteins. MyD88- and TRIFdependent activation of $N F-\kappa B$ signaling pathway was inhibited by basigin-specific antibody and siRNA. In addition, induction of TRIF-dependent, but MyD88-independent, AP-1 activation was inhibited by basigin-specific siRNA. Taken together, these results suggest that basigin plays a key regulatory role in activation of MyD88-and TRIF-mediated cell signaling pathways, and provide an important insight into basigin-mediated cellular processes that can serve as a guide for the development of new anti-inflammatory therapeutics via intervention in basigin-mediated cellular functions.
\end{abstract}

Keywords: Basigin, Signal transduction, $N F-\kappa B, M y D 88$, TRIF, AP-1

\section{Introduction}

Basigin/EMMPRIN/CD147, a member of the Ig superfamily, is a highly glycosylated plasma membrane protein involved in a variety of physiological functions, including regulation of lymphocyte responsiveness and monocarboxylate transporter expression, and an extracellular matrix metalloproteinase inducer (EMMPRIN) [1-3]. In cancer, overexpression of basigin has been shown to promote tumor progression and invasion by induction of various matrix metalloproteinases (MMPs) [4-6]. Basigin also enhances cell proliferation and vascular endothelial growth factor (VEGF) production by malignant melanoma cells, and promotes tumor cell glycolysis by facilitating lactate transport, in combination with monocarboxylate transporters, resulting in tumor progression and regulation of multidrug resistance (MDR) to anticancer drugs [7-9]. Recent work by Kang et al. indicated that basigin plays a key role in anticancer drug resistance in cancer stem-cell-like cells [10], implying that basigin could be an 
alternative target for overcoming drug resistance. Other in vitro and in vivo studies have suggested that basigin is involved in the regulation of inflammatory responses in a number of diseases, including rheumatoid arthritis [11-12], systemic lupus erythematosus [13], Alzheimer's disease [14], and acute lung disease [15].

Basigin is, thus, likely to be a major mediator of cellular processes. However, development of basigin-targeted therapy has been challenged by its diverse pathophysiological functions and its complicated interactions with other regulators and signaling pathways. The interaction between basigin and cyclophilin A, for example, initiates activation of the extracellular signal-regulated kinase (ERK) $1 / 2$ signaling cascade [16]. Basigin has also been shown to induce the expression of interferoninduced transmembrane protein 1 (IFITM1) through ERK and PIK in THP-1 cells [17]. The basigin-syndecan association induces activation of p44/42 mitogen-activated protein kinase (MAPK), which promotes cell adhesion and chemotaxis [18]. Recently, we reported that the basigin cascade promotes survival in cancer stem-cell-like cells through a Wnt $/ \beta$-catenin-dependent mechanism [10]. These data demonstrate that basigin plays a key regulatory role in signaling pathways, through association with extra- and intracellular molecules, in the regulation of inflammatory diseases and cancers. The adaptor proteins myeloid differentiation factor 88 (MyD88) and TIRdomain-containing adaptor-inducing interferon (TRIF) have been shown to play central roles in activation of $\mathrm{NF}-\kappa \mathrm{B}$ through Toll-like receptor (TLR) signaling transduction, which regulates cell proliferation, survival, and inflammation.

Herein, we have investigated the potential role of basigin in adaptor proteindependent activation of the NF- $\mathrm{KB}$ and AP-1 signaling pathways. We have shown that basigin induces activation of NF- $\kappa \mathrm{B}$ in a MyD88- and TRIF-dependent manner, and induces AP-1 activation in a TRIF-dependent manner. To our knowledge, this is the first report of the role of basigin in MyD88- and TRIF-mediated activation of signal transduction, and provides additional insights into the involvement of basigin in intracellular events.

\section{Materials and Methods}

\subsection{Flow cytometry}

HEK293T cells were maintained in DMEM containing 10\% FBS (Hyclone) and $100 \mu \mathrm{g} / \mathrm{mL}$ streptomycin-penicillin (Gibco). CHO cells were maintained in DMEM/F12 supplemented with $10 \%$ FBS, $0.1 \mathrm{mM}$ sodium hypoxanthine, $16 \mu \mathrm{M}$ thymidine, and $100 \mu \mathrm{g} / \mathrm{mL}$ streptomycin-penicillin. Endogenous cell-surface basigin expression was analyzed by flow cytometry using anti-basigin antibody (Genetex). Briefly, $1 \times 10^{6}$ cells were resuspended in $100 \mu \mathrm{L}$ ice-cold FACS buffer (PBS, $2 \% \mathrm{FBS}, 0.1 \% \mathrm{NaN}_{3}, \mathrm{pH} 7.4$ ) and incubated with $100 \mu \mathrm{L}$ of $10 \mu \mathrm{g} / \mathrm{mL}$ anti-basigin antibody for $45 \mathrm{~min}$ at $4{ }^{\circ} \mathrm{C}$. Cells were then washed with ice-cold FACS buffer three times by centrifugation at $300 \mathrm{x} g$ for $5 \mathrm{~min}$ and incubated with $200 \mu \mathrm{L}$ of secondary FITC-conjugated goat anti-mouse antibody (Jackson ImmunoResearch). Secondary and isotype-control antibodies were used as negative controls. Cells were washed with ice-cold FACS buffer as before and fixed with $0.5 \mathrm{~mL}$ of $0.5 \%$ paraformaldehyde in $\mathrm{PBS}, \mathrm{pH} 7.2$, for $15 \mathrm{~min}$ at $4{ }^{\circ} \mathrm{C}$ prior to analysis using a FACSCalibur flow cytometer (BD Biosciences) at the Central Laboratory of Kangwon National University. 


\subsection{Immunoblotting}

For detection of endogenous basigin by western analysis, whole-cell lysates were prepared in ice-cold cell lysis buffer $(25 \mathrm{mM}$ Tris- $\mathrm{HCl}, \mathrm{pH} 7.4,150 \mathrm{mM} \mathrm{NaCl}, 5 \mathrm{mM}$ EDTA, $1 \mathrm{mM}$ phenylmethylsulfonyl fluoride (PMSF), $1 \mu \mathrm{g} / \mathrm{mL}$ aprotinin, $1 \mu \mathrm{g} / \mathrm{mL}$ leupeptin, and $0.5 \%$ TritonX-100) and centrifuged at $12,000 \mathrm{x} \mathrm{g}$ for $15 \mathrm{~min}$ at $4{ }^{\circ} \mathrm{C}$. Total soluble protein was determined using the BCA Protein Assay Kit (Pierce). Equal amounts of soluble protein were loaded and resolved by SDS-PAGE under reducing conditions and transferred to a nitrocellulose membrane. The membrane was then blocked with $3 \%$ nonfat milk in TBS-T $(10 \mathrm{mM}$ Tris- $\mathrm{HCl}, 100 \mathrm{mM} \mathrm{NaCl}, 0.1 \%$ Tween 20, $\mathrm{pH} 7.5$ ) for $1 \mathrm{hr}$ at room temperature, and proteins were detected with $1 \mu \mathrm{g} / \mathrm{mL}$ anti-basigin antibody (GeneTex) followed by HRP-conjugated secondary antibody (Jackson ImmunoResearch) according to the manufacturer's protocols, and by reference to prestained molecular weight standard markers (Invitrogen). Immunoblots were visualized with the enhanced chemiluminescence (ECL) detection system (Pierce) and exposed to X-ray film.

\subsection{Luciferase reporter assay}

Luciferase constructs containing NF- $\mathrm{BB}-$ or AP-1-binding promoters and the reporter assay were as previously described [19]. Plasmids carrying wild-type MyD88 and TRIF were obtained from Addgene. Briefly, $4 \times 10^{5}$ subconfluent HEK293T cells in

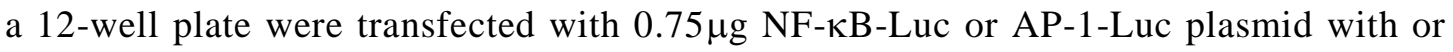
without $0.75 \mu \mathrm{g}$ MyD88 or TRIF together with $\beta$-galactosidase-Luc plasmid $(0.15 \mu \mathrm{g})$ as an internal control. After 6-hr incubation, medium was replaced with fresh complete medium, and cells were incubated overnight in a $\mathrm{CO}_{2}$ incubator. Cells were then treated with or without anti-basigin antibody (Genetex) for $8 \mathrm{hr}$, and cell lysates were prepared in ice-cold $10 \mathrm{mM} \mathrm{KH}_{2} \mathrm{PO}_{4} / 1 \mathrm{mM}$ EDTA and centrifuged at $12,000 \times \mathrm{g}$ for $5 \mathrm{~min}$ at $4{ }^{\circ} \mathrm{C}$. Luciferase activity was measured using the Luciferase Assay System (Promega) and VictorX4 luminometer (PerkinElmer). Experiments were carried out in quadruplicate for each sample, and luciferase activity was normalized to the $\beta$-galactosidase activity of the internal control.

\subsection{Small interfering RNA (siRNA)-directed basigin knockdown}

siRNA-mediated basigin silencing was carried out essentially as described for the luciferase reporter assay above, with the following modifications. Cells $\left(1 \times 10^{5}\right)$ were seeded in a 24-well plate, and transfected with $0.5 \mu \mathrm{g}$ each NF- $\kappa \mathrm{B}$ or AP-1 luciferase reporter gene and plasmid harboring MyD88 or TRIF with and without 400nM chemically synthesized siRNA (Genolution) targeting basigin, or negative control siRNA, in a complex with Lipofectamine (Invitrogen) according to the manufacturer's instructions. Cells were lysed, and luciferase activity was analyzed and normalized to $\beta$ galactosidase activity as described above.

\section{Result}

\subsection{Effect of basigin on activation of the MyD88-dependent NF- $\mathrm{KB}$ signaling pathway}

We first evaluated the two selected cell lines for endogenous basigin expression using flow cytometry and showed that basigin was readily detectible in HEK293 cells, but not in 

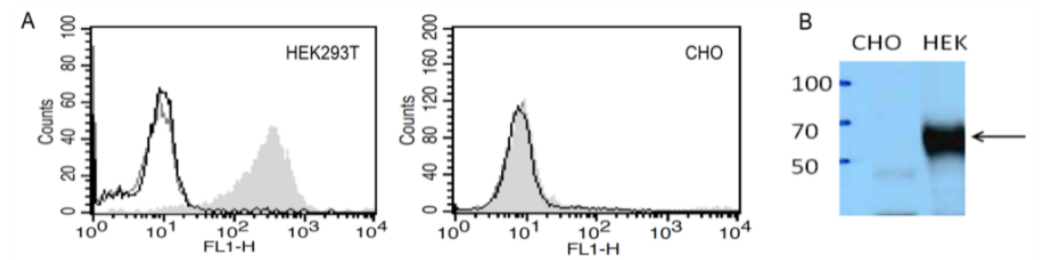

Figure 1. Analysis of endogenous basigin expression on the cell-surface of HEK293 and CHO by FACS $(A)$ and western blot $(B)$. Cells were stained with anti-basigin (shaded area) or isotype control antibody (gray line) followed by FITC labeled secondary antibody (black line), and proteins were detected using HRP labeled secondary antibody and TMB substrate

$\mathrm{CHO}$ cells (Figure 1A). The level of basigin expression in these cells was further confirmed by western analysis, which showed a high expression level in HEK293 cells, consistent with the results of FACS analysis (Figure 1B). Hence, subsequent studies of basigin-mediated signaling cascades associated with adaptor proteins reported herein were conducted using the HEK293 cell line.

To investigate whether basigin is involved in the transcriptional activation of NF- $\kappa \mathrm{B}$ with the MyD88 adaptor protein, cells were initially co-transfected with NF- $\kappa \mathrm{B}-$ luciferase reporter gene with MyD88 or a control plasmid, together with the $\beta$ galactosidase reporter as an internal control. Enhanced activity of $\mathrm{NF}-\kappa \mathrm{B}-$ driven luciferase activity was observed only in cells transfected with MyD88, and not in cells transfected with control vector, suggesting that MyD88 may be involved in activation of the NF- $\kappa$ B signal transduction pathway in HEK293 cells (Figure 2A).

We then investigated whether basigin affects MyD88-mediated NF- $\kappa \mathrm{B}$ activation using a basigin-specific antibody. As shown in Figure 2A, the addition of anti-basigin antibody resulted in inhibition of MyD88-mediated NF- $\kappa \mathrm{B}$ activation by $\sim 45 \%$. This suggests that basigin may mediate activation of NF- $\kappa \mathrm{B}$ through its association with MyD88 and/or that basigin and MyD88 act synergistically to activate NF- $\kappa$ B signaling. To further investigate a potential association between basigin and MyD88 leading to canonical NF- $\kappa \mathrm{B}$ activation, we analyzed the MyD88-mediated NF- $\kappa \mathrm{B}$ luciferase activity
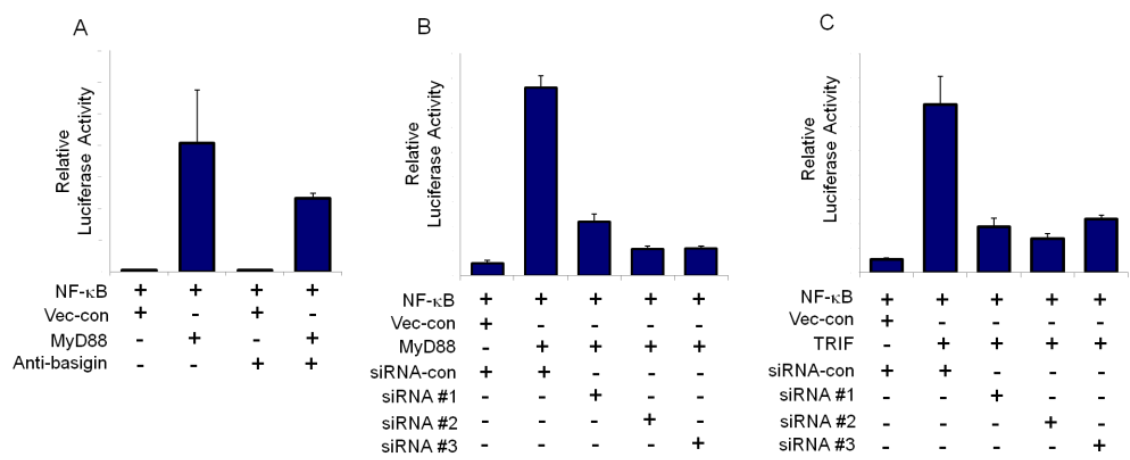

Figure 2. Effect of basigin on MyD88-induced ( $A$ and $B$ ) and TRIF-induced $(C)$ NF- $\square$ B activation using anti-basigin antibody $(A)$ and basigin-specific siRNA ( $B$ and $C$ ). Values are mean ${ }_{ \pm} S E M$, and are representative of three independent experiments. Empty parent vector was used as a negative control 

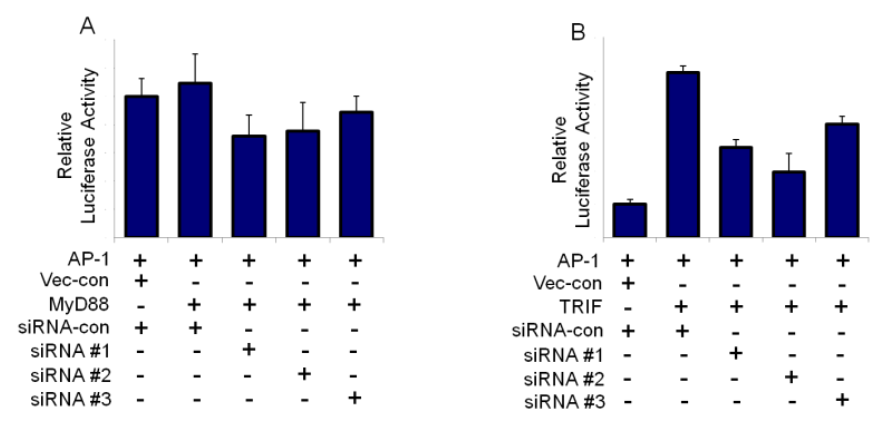

Figure 3. Effect of basigin on AP-1 activation mediated by MyD88 $(A)$ and TRIF $(B)$. Values are mean ${ }_{ \pm} S E M$, and are representative of three independent experiments

Following knockdown of basigin using three different basigin-specific siRNAs. Interestingly, approximately $85 \%$ inhibition of $\mathrm{NF}-\mathrm{\kappa B}$-driven luciferase activity was observed with basigin knockdown, whereas the control siRNA had no effect on MyD88mediated NF- $\kappa \mathrm{B}$ activation (Figure $2 \mathrm{~B}$ ). Taken together, these results indicate that basigin regulates NF- $\kappa \mathrm{B}$ activation through MyD88 signaling.

\subsection{Effect of basigin on activation of the TRIF-dependent NF- $\mathrm{kB}$ signaling pathway}

Because TRIF is also known to associate with TLR families, we further investigated the involvement of basigin in the TRIF-mediated NF- $\kappa B$ signaling cascade in HEK293 cells cotransfected with NF- $\kappa \mathrm{B}$-driven luciferase reporter with and without TRIF. Strong induction of NF- $\kappa \mathrm{B}$-driven luciferase activity by TRIF, comparable to the corresponding activation by MyD88, was observed (Figure 2C). Additionally, TRIFinduced NF- $\kappa \mathrm{B}$ luciferase activity was blocked by the basigin-specific siRNAs to an extent similar to that shown for MyD88 in Figure 2B, which suggests that basigin also mediates TRIF-dependent NF- $\kappa \mathrm{B}$ activation.

\subsection{Effect of basigin on activation of the TRIF-dependent AP-1 signaling pathway}

Recently, Rac-1-dependent activation of AP-1 signaling by basigin was also reported [20]. Hence, we investigated whether AP-1 activation is a MyD88- or TRIF-dependent event in the basigin-mediated signaling transduction pathway. As shown in Figure 3A, no significant difference in AP-1-driven luciferase activity was observed between cells transfected with and without MyD88, suggesting that AP-1 signaling is independent of MyD88. However, when the cells were treated with basigin-specific siRNAs, AP-1 activity was reduced by $\sim 30 \%$, suggesting potential inhibition of intrinsic AP-1 activation by basigin knockout. In contrast to MyD88, strong enhancement of AP-1 activation by TRIF was observed (Figure 3B), whereas the basigin-specific siRNA treatment significantly reduced the TRIF induced AP-1 activity (Figure 3B). This observation indicates that basigin is also involved in regulation of the TRIF-mediated AP-1 activation pathway.

\section{Discussion}

Basigin has been emerging as a key regulatory protein involved in major pathophysiological processes, underlying conditions such as cancer and inflammatory 
disease, and has received attention as a promising therapeutic target. Several mechanisms of basigin regulation through extracellular and intracellular interactions with functional molecules have been reported. Evaluation of several signaling pathways has demonstrated an association between basigin and the p38 MAPK cascade and activation of ERK cascades [17]. Recently, we also demonstrated that suppression of basigin expression results in activation of GSK3 and down regulation of $\mathrm{Wnt} / \beta$-catenin, demonstrating the regulatory role of basigin in the $\mathrm{Wnt} / \beta$-catenin signaling pathway that confers anti-cancer drug resistance [10]. Although current knowledge of mechanisms of basigin action suggests that basigin plays a key pathophysiological role in many diseases and, thus, represents a promising therapeutic target, the precise molecular mechanism by which basigin mediates its effects has not been fully elucidated.

In this study, we report that the adaptor proteins MyD88 and TRIF are functionally associated with basigin. Investigation of a potential association between basigin and MyD88 in an NF- $\kappa \mathrm{B}$-directed luciferase assay using basigin-specific neutralizing antibody or siRNAs showed that basigin mediates NF- $\kappa \mathrm{B}$ activation through a functional association with MyD88. MyD88 is known to be associated with the TLR and interleukin-1 receptor (IL-1R) superfamily involved in activation of NF- $\kappa \mathrm{B}$ and MAPK. Our observation suggests that association of basigin and MyD88 clearly leads to the canonical pathway of NF- $\kappa \mathrm{B}$ activation.

In addition to MyD88, TRIF is an adaptor protein that appears to participate in basigin-mediated canonical NF- $\kappa \mathrm{B}$ activation, as evidenced by the reduction in TRIFmediated NF- $\kappa$ B activation by basigin-specific siRNA. TRIF was also found to be involved in a MyD88-independent TLR signaling pathway. Both in vitro and in vivo studies have demonstrated that TRIF is involved in the production of inflammatory cytokines in response to TLR4 ligand binding and suggested that TRIF is essential for TLR3- and TLR4-mediated signaling pathways, which facilitate mammalian antiviral host defense [21]. We proposed that TRIF is an additional adaptor protein associated with basigin-mediated canonical NF- $\kappa \mathrm{B}$ activation. Taken together, our results support the hypothesis that basigin is associated with MyD88- and TRIF-mediated activation of signaling pathways. However, further studies are needed to evaluate whether MyD88 and TRIF interact cooperatively with basigin or other regulators of signaling pathways. In addition to basigin-directed NF- $\mathrm{BB}$ activation, AP-1 activation is also triggered through basigin in a TRIF-dependent, but MyD88-independent, manner, as reported for the TLR signaling pathway [21]. The present study provides the first evidence that basigin is coordinately associated with AP-1 activation in a TRIF-dependent manner. Coordinated activity of AP-1 and NFAT in gene regulation has been reported [22]. Likewise, further studies are needed to identify other regulators or signaling pathways that are co-operatively associated with AP-1.

In summary, we have provided evidence that endogenous basigin-mediated NF- $\kappa \mathrm{B}$ activation takes place in a MyD88- and TRIF-dependent manner. Further studies are underway to investigate whether MyD88 and TRIF cooperate with basigin and if regulators in other signaling pathways are involved. We also hypothesized that the basigin signaling cascade may intersect with other TLR signaling pathways. The TRIFdependent basigin/AP-1 signaling cascade is another area for further investigation. The findings of this study provide new insight into basigin-mediated cell functions, which may guide the strategies for targeting basigin as a promising inflammatory disease or cancer therapeutic. 


\section{Acknowledgements}

This work was supported by NRF-2011-0025320 and the Proteogenomic Research Program. Flow cytometric analysis was conducted at the Central Laboratory of Kangwon National University. The authors declare no financial or commercial conflict of interest.

\section{References}

[1] P. Kirk, M. C. Wilson, C. Heddle, M. H. Brown, A. N. Barclay and A. P. Halestrap, EMBO J, vol. 19, (2000), pp. 3809.

[2] C. Biswas, Y. Zhang, R. DeCastro, H. Guo, T. Nakamura, H. Kataoka and K. Nabeshima, Cancer Res., vol. 55, (1995), pp. 434.

[3] H. Guo, S. Zucker, M. K. Gordon, B. P. Toole and C. Biswas, J. Biol. Chem., vol. 272, (1997), pp. 24.

[4] B. Davidson, V. Givant-Horwitz, P. Lazarovici, B. Risberg, J. M. Nesland, C. G. Trope, E. Schaefer and R. Reich, Clin. Exp. Metastasis, vol. 20, (2003), pp. 621.

[5] B. Davidson, I. Goldberg, R. Reich, L. Tell, H.P. Dong, C.G. Trope, B. Risberg and J. Kopolovic, Gynecol. Oncol., vol. 90, (2003), pp. 248.

[6] T. Kanekura, X. Chen and T. Kanzaki, Int. J. Cancer, vol. 99, (2002), pp. 520.

[7] Y. Tang, M.T. Nakada, P. Kesavan, F. McCabe, H. Millar, P. Rafferty, P. Bugelski and L. Yan, Cancer Res., vol. 65, (2005), pp. 3193.

[8] H. Chen, L. Wang, J. Beretov, J. Hao, W. Xiao and Y. Li, Clin. Exp. Metastasis, vol. 27, (2010), pp. 557.

[9] L. Jia, H. Xu, Y. Zhao, L. Jiang, J. Yu and J. Zhang, Cancer Invest., vol. 26, (2008), pp. 977.

[10] M. J. Kang, H. P. Kim, K. S. Lee, Y. D. Yoo, Y. T. Kwon, K. M. Kim, T. Y. Kim and E. C. Yi, Proteomics, vol. 13, (2013), pp. 1714.

[11] T. Tomita, T. Nakase, M. Kaneko, K. Shi, K. Takahi, T. Ochi and H. Yoshikawa, Arthritis Rheum., vol. 46, (2002), pp. 373.

[12] Y. T. Konttinen, T. F. Li, J. Mandelin, M. Liljestrom, T. Sorsa, S. Santavirta and I. Virtanen, Arthritis Rheum., vol. 43, (2000), pp. 275.

[13] G. Pistol, C. Matache, A. Calugaru, C. Stavaru, S. Tanaseanu, R. Ionescu, S. Dumitrache and M. Stefanescu, J. Cell Mol. Med., vol. 11, (2007), pp. 339.

[14] J. Nahalkova, I. Volkmann, M. Aoki, B. Winblad, N. Bogdanovic, L.O. Tjernberg and H. Behbahani, Neurochem. Int., vol. 56, (2010), pp. 67.

[15] H. D. Foda, E. E. Rollo, M. Drews, C. Conner, K. Appelt, D. R. Shalinsky and S. Zucker, Am. J. Respir. Cell Mol. Biol., vol. 25, (2001), pp. 717.

[16] K. Kim, H. Kim, K. Jeong, M. H. Jung, B. S. Hahn, K. S. Yoon, B. K. Jin, G. H. Jahng, I. Kang, J. Ha and W. Choe, Apoptosis, vol. 17, (2012), pp. 784.

[17] J. Y. Kim, H. Kim, K. Suk and W. H. Lee, Mediators Inflamm., vol. 2010, (2010), pp. 821940.

[18] R. Pakula, A. Melchior, A. Denys, C. Vanpouille, J. Mazurier and F. Allain, Glycobiology, vol. 17, (2007), pp. 492.

[19] J. E. Jeong, E. G. Kim, J. Y. Cho, E. C. Yi and K. M. Kim, Advanced Science and Technology Letters, vol. 33, (2013), pp. 20.

[20] B. Venkatesan, A. J. Valente, S. D. Prabhu, P. Shanmugam, P. Delafortaine and B. Chandrasekar, J. Mol. Cell Cardiol., vol. 49, (2010), pp. 655.

[21] M. Yamamoto, S. Sato, H. Hemmi, K. Hoshino, T. Kaisho, H. Sanjo, O. Takeuchi, M. Sugiyama, M. Okabe, K. Takeda and S. Akira, Science, vol. 301, (2003), pp. 640.

[22] F. Macian, C. Lopez-Rodriguez, and A. Rao, Oncogene, vol. 20, (2001), pp. 2476. 


\section{Authors}

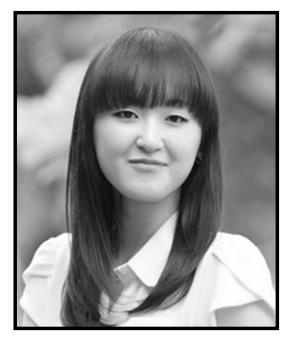

\section{Jieun Jeong}

She received the B.S. degree in medical biotechnology from Kangwon National University in 2012 and the Ph.D. candidate in Systems Immunology.

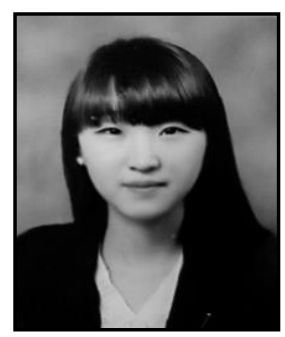

\section{Eunhee Grace Kim}

She received the B.S. degree in medical biotechnology from Kangwon National University in 2012 and the Ph.D. candidate in Systems Immunology.

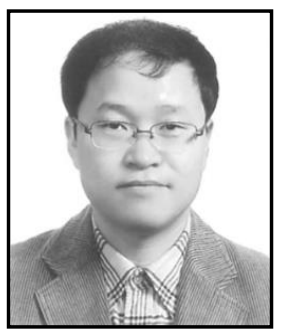

\section{Jae Y. Cho}

She received the B.S. and M.S. degree from Sungkyunkwan University in 1991 and 1993, respectively. He received the Ph.D. degreee in Immunology from University of College London in 2001. He is an associate professor at the Sungkyunkwan University. His research interests include TLR related signal transduction pathway and development of biomaterials for inflammatory diseases.

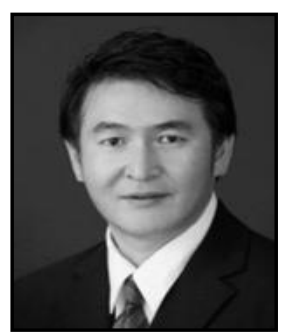

\section{Eugene C. Yi}

He received the B.S. degree in Chemsitry from Washington State University and a Ph.D. degreee in Biological Chemistry from the University of Utah in 1996. After his post-doctoral research fellowship at Howard Hughes Medical Institute, University of Michigan, he joined Institute for Systems Biology in Seattle as a scientist to develop proteomc technologies and their application in vaious biological systems. He then moved to ZymoGentics (Bristol Myer Squibb) and Novo Nordisk, and implemented proteomic platforms in support of therapeutic target discovery in cancer and autoimmune diseases. In 2009, he joined Seoul National University as professor. The focus of his research at the university is the development and implementation of translational-focused proteomic tools for clinical research related to cancer and autoimmune/inflammatory diseases. 


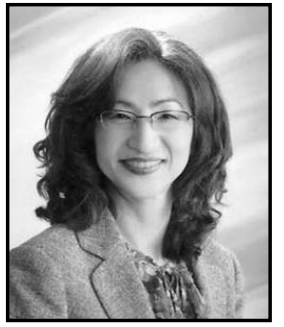

\section{Kristine M. Kim}

She received the B.S. degree in Biochemistry from Washington State University and a Ph.D. degreee in Biological Chemistry from the University of Utah in 1996. Following her post-dotoral research fellowship at the University of Michgan, she worked on Bcl-2 proteins involved in the programmed cell death at Fred Hutchinson Cancer Research Center, and the developmen of therapeutic antibody at Immunex/Amgen and Seattle Genetics. She is an associate professor at the Kangwon National University. Her current research interests include therapeutic target discovery, elucidating mechanisms of drug resistances and development of therapeutic antibodies and antibody-drug conjugates (ADCs) for cancer and inflammatory diseases. 
International Journal of Bio-Science and Bio-Technology Vol.6, No.2 (2014) 